https://doi.org/10.15407/ujpe64.5.380

YU.V. KOVTUN, A.I. SKIBENKO, E.I. SKIBENKO, YE.V. SIUSKO

National Science Center "Kharkiv Institute of Physics and Technology"

(1, Akademichna Str., Kharkiv 61108,Ukraine; e-mail: Ykovtun@kipt.kharkov.ua)

\title{
SPECIFIC FEATURES OF MICROWAVE METHODS FOR DUSTY PLASMA DIAGNOSTICS. I. DIELECTRIC PERMITTIVITY, REFRACTIVE AND ABSORPTION INDICES
}

\begin{abstract}
Two widely used approaches for the determination of the refractive, $n$, and absorption, $\varkappa$, indices of a dusty plasma have been analyzed. In one of them, the expressions for $n$ and $\varkappa$ obtained for a dust-free plasma are used, but the collisions of plasma ions with dust particles are taken into account by means of the collision frequency parameter. In the other approach, the characteristic charging frequency for dust particles is additionally introduced.

Ke y words: dusty plasma, refractive index, absorption index, microwave diagnostic methods, dielectric permittivity.
\end{abstract}

\section{Introduction}

The progress and development of researches in physics of plasma, including a high-temperature plasma of controlled fusion reactions, are governed, to a large extent, by the innovations implemented in the methods for measuring plasma parameters. Among the methods of plasma diagnostics, microwave methods [1-4] are widely used nowadays. Their advantage consists in their contactless character and the absence of restrictions on the plasma temperature.

Both active and passive methods are used in the plasma diagnostics. When using the passive method, the own microwave radiation emitted by a plasma is measured. On the other hand, the interaction of electromagnetic waves with a plasma and the measurement of the results of this interaction form the basis of active methods.

Among the active methods that are most often used in the plasma diagnostics, there are methods that are based on the measurement of the phase shift or ab-

(c) YU.V. KOVTUN, A.I. SKIBENKO, E.I. SKIBENKO, YE.V. SIUSKO, 2019 sorption index for an electromagnetic wave that propagates through a plasma; e.g., this is the method of plasma interferometry. In this method, the measurement results are used to determine the refractive index $n$ and the absorption index $\varkappa[1-4]$ :

$\varphi_{p}=\frac{\omega}{c} \int_{0}^{S_{p}} n(s) d s$,
$\mu_{p}=\frac{\omega}{c} \int_{0}^{S_{p}} \varkappa(s) d s$,

where $\omega$ is the probing frequency, $c$ the light speed, $n(s)$ and $\varkappa(s)$ are the refractive and absorption, respectively, indices at the given point $s$ in the plasma, and $S_{p}$ is the path of a microwave beam in the plasma.

The active methods are used to measure some parameters in both weakly and strongly ionized plasma (the electron concentration, the collision frequency, and others), as well as in a high-temperature plasma in experiments dealing with controlled thermonuclear

ISSN 2071-0194. Ukr. J. Phys. 2019. Vol. 64, No. 5 
fusion. Recently, the application scope of active microwave methods was somewhat extended owing to their usage in the diagnostics of a dusty plasma [5-8].

The dusty plasma (complex, colloidal plasma) is an ionized gas that contains macroscopic (micronand submicron-sized) charged particles [9-15]. The interest in a dusty plasma is associated with its wide spread in the nature (atmosphere, outer space, and so forth) and in technological plasma installations (etching, sputtering, thermonuclear, and others). The research of the processes running in a dusty plasma and the determination of its properties can be useful for the solution of many physical and applied problems. The study of the dusty plasma properties showed that dust particles can form plasma clusters and crystals. As a rule, plasma particles (macroscopic particles) are charged negatively, and their charge can reach a value of up to $10^{5}$ times the electron charge. The presence of charged dust particles not only changes the balance between the electron and ion concentrations, thus affecting the propagation of plasma oscillations, but also results in the emergence of new low-frequency waves [9-15].

One of the specific features of a dusty plasma is the influence of dust particles on the scattering of electromagnetic waves in the plasma. In works [1620 ], it was shown that the scattering of electromagnetic waves depends on the following parameters: the concentration, size, and charge of dust particles. In works $[21,22]$, the influence of dust particles on the scattering of electromagnetic waves under ionospheric conditions was considered.

As was already mentioned, the development of microwave methods for the dusty plasma diagnostics has received an impetus in recent years [5-8]. There are several approaches aimed at the consideration of the interaction between electromagnetic waves and a dusty plasma [23, 24]. Rather often, this consideration includes issues that go beyond the scope of the microwave application for the dusty plasma diagnostics. The literature on this issue is scarce and occasional.

The aim of this work is to analyze the method used, while considering the interaction of microwaves with a dusty plasma, namely, to summarize the corresponding literature data, and to consider the scope of applications and the specific features of active microwave methods used for the dusty plasma diagnostics.

\section{Propagation of Electromagnetic Waves Through a Plasma}

\subsection{Dielectric permittivity of a plasma}

In the general case, the complex dielectric permittivity of a medium can be written in the form $[25,26]$

$\varepsilon^{\prime}{ }_{i j}(\omega, k)=\delta_{i j}+\sum_{a} \chi_{i j}^{(a)}(\omega, k)$,

where $\delta_{i j}$ is the identity tensor,

$\chi_{i j}^{(a)}(\omega, k)=i \frac{\sigma_{i j}^{(a)}(\omega, k)}{\omega \epsilon_{0}}$

is the tensor of high-frequency dielectric susceptibility (polarizability) of the $a$-th plasma component, $\sigma_{i j}^{(a)}(\omega, k)$ the complex conductivity tensor, $\omega$ the frequency, $k$ the wave vector, and $\epsilon_{0}$ the electric constant. The dependences of the indicated tensors on the frequency $\omega$ and on the wave vector $k$ are responsible, respectively, for the frequency dispersion and the spatial dispersion of the electromagnetic field in the medium. If the velocity distribution of plasma particles is isotropic, the tensor $\chi_{i j}^{(a)}(\omega, k)$ can be expressed only in terms of the identity tensor $\delta_{i j}$ and the tensor $k_{i} k_{j}$. Accordingly, for an isotropic medium, the tensor $\chi_{i j}^{(a)}(\omega, k)$ can be expressed in the form

$\varepsilon^{\prime}{ }_{i j}(\omega, k)=\left(\delta_{i j}-\frac{k_{i} k_{j}}{k^{2}}\right) \varepsilon^{t r}(\omega, k)+\frac{k_{i} k_{j}}{k^{2}} \varepsilon^{l}(\omega, k)$,

where $\varepsilon^{t r}(\omega, k)$ and $\varepsilon^{l}(\omega, k)$ are the transverse and longitudinal, respectively, dielectric permittivities. In the case of a cold plasma, an insignificant influence of the particle thermal motion on the wave propagation and the spatial dispersion can be neglected. Accordingly, we obtain the following equations:

$\varepsilon^{t r}(\omega, k)=\varepsilon^{l}(\omega, k)=\varepsilon^{\prime}(\omega), \varepsilon_{i j}^{\prime}=\varepsilon^{\prime}(\omega) \delta_{i j}$.

In the absence of an external magnetic field, a cold plasma is isotropic. In this case, the complex dielectric permittivity is determined as follows [27]:

$\varepsilon^{\prime}=\varepsilon-i \frac{\sigma}{\omega \epsilon_{0}}$

where $\varepsilon$ is the dielectric permittivity, and $\sigma$ the highfrequency plasma conductivity. In the general case, the parameters $\varepsilon^{\prime}, \varepsilon$, and $\sigma$ depend on the frequency and the coordinates. 
For a two-component plasma containing electrons and ions, the quasineutrality condition looks like

$N_{e}=Z_{i} N_{i}$

where $N_{e}$ is the electron concentration in a plasma, $N_{i}$ the ion concentration, and $Z_{i}$ the charge number of ions. Accordingly, the dielectric permittivity $\varepsilon$ and the conductivity $\sigma$ of a plasma are determined by the formulas [27]

$$
\begin{aligned}
& \varepsilon=1-\frac{N_{e} e^{2}}{\epsilon_{0} m_{e}\left(\omega^{2}+\nu_{(e), \mathrm{eff}}^{2}\right)}-\frac{N_{i} Z_{i}^{2} e^{2}}{\epsilon_{0} m_{i}\left(\omega^{2}+\nu_{(i), \mathrm{eff}}^{2}\right)}, \\
& \sigma=\frac{N_{e} e^{2} \nu_{(e), \mathrm{eff}}}{m_{e}\left(\omega^{2}+\nu_{(e), \mathrm{eff}}^{2}\right)}+\frac{N_{i} Z_{i}^{2} e^{2} \nu_{(i), \mathrm{eff}}}{m_{i}\left(\omega^{2}+\nu_{(i), \mathrm{eff}}^{2}\right)},
\end{aligned}
$$

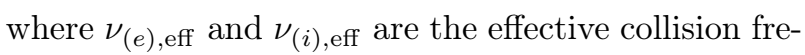
quencies of electrons and ions, respectively, with dust particles; and $m_{e}$ and $m_{i}$ are the electron and ion, respectively, masses. Since the mass ratio $m_{e} / m_{i} \ll$ $\ll 1$ - e.g., for the hydrogen atom (proton), we have $m_{e} / m_{i}=5.446 \times 10^{-4}-$ the influence of ions is very small, as a rule, and it is not taken into consideration, as usual. The account for ions is required, if the concentration of negative ions in a plasma is high or the concentration of electrons is very low. If the ionic contribution is neglected, Eqs. (7) and (8) take the form

$$
\begin{aligned}
& \varepsilon=1-\frac{N_{e} e^{2}}{\epsilon_{0} m_{e}\left(\omega^{2}+\nu_{\text {eff }}^{2}\right)}, \\
& \sigma=\frac{N_{e} e^{2} \nu_{\text {eff }}}{m_{e}\left(\omega^{2}+\nu_{\text {eff }}^{2}\right)},
\end{aligned}
$$

where

$\nu_{\mathrm{eff}}=\nu_{e i}+\nu_{\text {en }}$

is the effective collision frequency of electrons with ions and neutral particles,

$\nu_{e i}=\frac{\sqrt{2} N_{i} Z_{i}^{2} e^{4} \Lambda_{e i}}{12 T_{e}^{3 / 2} m_{e}^{1 / 2} \pi^{3 / 2} \epsilon_{0}^{2}}$

is the frequency of electron-ion collisions, $\Lambda_{e i}$ the Coulomb logarithm,

$\nu_{e n}=N_{n} K_{e l}$

is the frequency of electron-neutral collisions, and $K_{e l}$ is the rate constant for electron-neutral collisions. Collisions between electrons in isotropic plasma are insignificant, when $\omega^{2} \gg \nu_{\text {eff }}^{2}$, so that they can be neglected [27].

\subsection{Refractive and absorption indices}

In the microwave plasma diagnostics, the dielectric permittivity and conductivity parameters play rather an auxiliary role. It is the refractive index $n$ and the absorption index $\varkappa$ that are directly determined. A relation of the complex dielectric constant with the indices $n$ and $\varkappa$ is given by the formula

$\varepsilon^{\prime}=(n-i \varkappa)^{2}$.

With regard for Eqs. (5) and (12) for $n$ and $\varkappa$, respectively, we obtain

$\varepsilon=n^{2}-\varkappa^{2}$,

$\frac{\sigma}{\omega \epsilon_{0}}=2 n \varkappa$.

From whence, in view of Eqs. (9) and (10), the refractive index $n$ and the absorption index $\varkappa$ can be written in the form [27]

$n^{2}=\frac{1}{2}\left(1-\frac{\omega_{p e}^{2}}{\omega^{2}+\nu_{\text {eff }}^{2}}\right)+$

$+\frac{1}{2} \sqrt{\left(1-\frac{\omega_{p e}^{2}}{\omega^{2}+\nu_{\mathrm{eff}}^{2}}\right)^{2}+\left(\frac{\omega_{p e}^{2}}{\omega^{2}+\nu_{\mathrm{eff}}^{2}} \frac{\nu_{\mathrm{eff}}}{\omega}\right)^{2}}$,

$\varkappa^{2}=-\frac{1}{2}\left(1-\frac{\omega_{p e}^{2}}{\omega^{2}+\nu_{\mathrm{eff}}^{2}}\right)+$

$+\frac{1}{2} \sqrt{\left(1-\frac{\omega_{p e}^{2}}{\omega^{2}+\nu_{\mathrm{eff}}^{2}}\right)^{2}+\left(\frac{\omega_{p e}^{2}}{\omega^{2}+\nu_{\mathrm{eff}}^{2}} \frac{\nu_{\mathrm{eff}}}{\omega}\right)^{2}}$,

where

$\omega_{p e}=\left(\frac{N_{e} e^{2}}{\epsilon_{0} m_{e}}\right)^{1 / 2}$.

is the electron plasma frequency. In the limiting case of high frequencies, i.e. if $\omega^{2} \gg \nu_{\text {eff }}^{2}$, Eqs. (9), (10), (15), and (16) can be somewhat simplified [27]:

$\varepsilon=1-\frac{N_{e} e^{2}}{\epsilon_{0} m_{e} \omega^{2}}$,

$\sigma \approx \frac{N_{e} e^{2} \nu_{\mathrm{eff}}}{m_{e} \omega^{2}}$

$n^{2}=\frac{1}{2}\left(1-\frac{\omega_{p e}^{2}}{\omega^{2}}\right)+$

$+\frac{1}{2} \sqrt{\left(1-\frac{\omega_{p e}^{2}}{\omega^{2}}\right)^{2}+\left(\frac{\omega_{p e}^{2}}{\omega^{2}} \frac{\nu_{\mathrm{eff}}}{\omega}\right)^{2}}$,

ISSN 2071-0194. Ukr. J. Phys. 2019. Vol. 64, No. 5 
$\varkappa^{2}=-\frac{1}{2}\left(1-\frac{\omega_{p e}^{2}}{\omega^{2}}\right)+$

$+\frac{1}{2} \sqrt{\left(1-\frac{\omega_{p e}^{2}}{\omega^{2}}\right)^{2}+\left(\frac{\omega_{p e^{2}}}{\omega^{2}} \frac{\nu_{\mathrm{eff}}}{\omega}\right)^{2}}$.

In the particular case where $\omega^{2} \gg \nu_{\text {eff }}^{2}$ and $\varepsilon \gg$ $\gg \sigma /\left(\omega \epsilon_{0}\right)(\varepsilon>0)$, Eqs. (19) and (20) for the refractive index $n$ and the absorption index $\varkappa$ can also be strongly simplified to the form [27]

$n \approx \sqrt{\varepsilon}=\left(1-\frac{\omega_{p e}^{2}}{\omega^{2}}\right)^{1 / 2}$

$\varkappa \approx \frac{1}{2} \frac{\sigma}{\omega \epsilon_{0} \sqrt{\varepsilon}}=\frac{1}{2} \frac{\omega_{p e}^{2}}{\omega^{2}} \frac{\nu_{\mathrm{eff}}}{\omega}\left(1-\frac{\omega_{p e}^{2}}{\omega^{2}}\right)^{-1 / 2}$.

Most often, expressions (21) and (22) are used, when the methods of microwave plasma diagnostics are considered and applied [1-4]. In the case where $\omega^{2} \gg \nu_{\text {eff }}^{2}$ and $\omega_{p e} \geq \omega$, the electromagnetic wave rapidly decays in plasma. If the penetration depth of the alternating field into a plasma is smaller than the thickness of a plasma with $\omega_{p e} \geq \omega$, then the electromagnetic wave becomes reflected from the plasma boundary. Figure 1 illustrates the dependences of the refractive (panel $a$ ) and absorption (panel $b$ ) indices on the ratio $\omega_{p e} / \omega$, which were calculated according to formulas (15) and (16).

\section{Propagation of Electromagnetic Waves in a Dusty Plasma}

\subsection{Dusty plasma}

The dusty plasma contains macroscopically small charged particles. In a gas-discharge plasma, provided that the emission processes are absent, a dust particle captures electrons and ions, so that it becomes electrically charged. Since the mobility of electrons is higher, the dust particle acquires a negative charge [9-15]. Owing to the processes of electron emission (thermionic, photoelectron, secondary electron emission) from the surface of a dust particle, its total charge decreases by magnitude and, under certain conditions, it may became positive. In this case, the coexistence of particles and electrons emitted by them in the framework of a common twocomponent system is possible. The account for dust
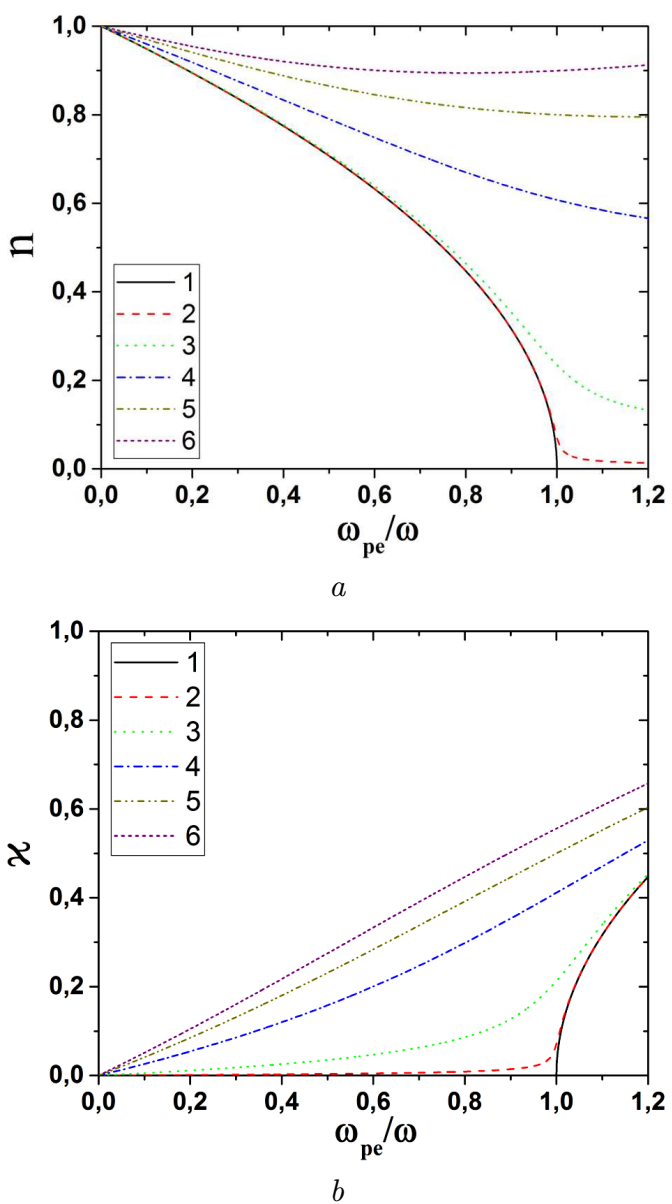

Fig. 1. Dependences of the refractive $(a)$ and absorption $(b)$ indices in a dusty plasma on the ratio $\omega_{p e} / \omega$ for various values of the ratio $\nu_{\text {eff }}^{2} / \omega^{2}=10^{-5}(1), 10^{-2}$ (2), 0.1 (3), 0.5 (4), 0.8 (5), and $1(6)$

particles leads to the following condition of plasma quasineutrality:

$N_{e}+Z_{d} N_{d}=Z_{i} N_{i}$,

where $N_{d}$ is the concentration of dust particles, and $Z_{d}$ their charge number.

In the orbital-motion-limited (OML) approximation, the cross-sections of the electron and singlecharged ion capture by a dust particle are determined by the following expressions [11]:

- for the electron capture,

$\sigma_{e}\left(v_{e}\right)= \begin{cases}0 & \text { if } \frac{2 e \phi_{s}}{m_{e} v_{e}^{2}} \leq-1, \\ \pi r_{d}^{2}\left(1+\frac{2 e \phi_{s}}{m_{e} v_{e}^{2}}\right) & \text { if } \frac{2 e \phi_{s}}{m_{e} v_{e}^{2}}>-1\end{cases}$ 
- for the ion capture,

$\sigma_{i}\left(v_{i}\right)=\pi r_{d}^{2}\left(1-\frac{2 e \phi_{s}}{m_{i} v_{i}^{2}}\right)$

where $r_{d}$ is the dust particle radius (most often, the dust particle is assumed to be spherical); $v_{e}$ and $v_{i}$ are the electron and ion, respectively, velocities with respect to the particle; and $\phi_{s}$ is the surface potential of the particle with respect to the plasma (it is adopted to be negative, $\left.\phi_{s}<0\right)$.

The stationary charge of a particle, $Z_{d}$, corresponds to the equality between the electron and ion fluxes absorbed by the particle. In the dimensionless form, it can be determined from the relation [11]

$\exp (-z)=\frac{N_{i}}{N_{e}}\left(\frac{\mu}{\tau}\right)^{1 / 2}(1+z \tau)(1+P)$,

where $\tau=T_{e} / T_{i}$ is the ratio between the electron and ion temperatures, $\mu=m_{e} / m_{i}$ is the ratio between the electron and ion masses,

$z=\frac{\left|Z_{d}\right| e^{2}}{r_{d} T_{e}}$

is the normalized absolute value of the dust particle charge, and

$P=\left|Z_{d}\right| \frac{N_{d}}{N_{e}}$

is the Havnes parameter determining the ratio between the product of the concentration and charge of dust particles, on the one hand, and the concentration of the electron component, on the other hand.

The charging frequency of a dust particle is defined as the relaxation frequency of small charge perturbations to the stationary value [11],

$v_{\mathrm{ch}}=\frac{1+z}{\sqrt{2 \pi}} \frac{r_{d}}{\lambda_{d i}} \omega_{p i}$,

where

$\lambda_{d i}=\left(\frac{\epsilon_{0} T_{i}}{N_{i} Z_{i}^{2} e^{2}}\right)^{1 / 2}$

is the ion Debye radius, and

$\omega_{p i}=\left(\frac{N_{i} Z_{i}^{2} e^{2}}{\epsilon_{0} m_{i}}\right)^{1 / 2}$

is the ion-plasma frequency.
In a dusty plasma, besides the electron-ion, $\nu_{e i}$, electron-neutral, $\nu_{e n}$, ion-ion, $\nu_{i i}$, and ion-neutral, $\nu_{i n}$, collision frequencies, the frequencies of collisions between dust particles and electrons, $\nu_{e d}$, ions, $\nu_{i d}$, and neutral atoms (molecules), $\nu_{n d}$, as well as between the particles themselves, $\nu_{d d}$, have to be taken into consideration [11]. The frequency of collisions between electrons and dust particles can be determined from the relation [28]

$\nu_{e d}=\frac{8 \sqrt{2 \pi}}{3} r_{d}^{2} N_{d} v_{e} \Phi_{e}(z, L)$,

where the multiplier $\Phi_{e}(z, L)$ makes allowance for the electrostatic interaction between an electron and a dust particle. For an uncharged particle, $\Phi_{e}(z, L)=$ $=1$. More specifically, this quantity involves the absorption and scattering of electrons by the dust particle:

$\Phi_{e}(z, L)=\Phi_{e}^{\text {coll }}(z)+\Phi_{e}^{o r b}(z, L)$,

where

$\Phi_{e}^{\text {coll }}(z)=\left(1+\frac{z}{2}\right) \exp (-z)$

and

$\Phi_{e}^{\mathrm{orb}}(z, L)=\frac{1}{4} z^{2} \Lambda_{e d}$.

For the electron-particle collisions, the Coulomb logarithm $\Lambda_{e d}$ is determined by the formula [28]:

$$
\begin{aligned}
& \Lambda_{e d}=\int_{0}^{\infty} e^{-x} \ln \left(1+4 L^{2} \frac{x^{2}}{z^{2}}\right) d x- \\
& -2 \int_{z}^{\infty} e^{-x} \ln \left(\frac{2 x}{z}-1\right) d x,
\end{aligned}
$$

where $L=\lambda_{d} / r_{d}$ is the ratio between the screening length (the Debye radius) and the particle radius. For the first parameter, we have

$\lambda_{d}=\frac{\lambda_{d e} \lambda_{d i}}{\left(\lambda_{d e}^{2}+\lambda_{d i}^{2}\right)^{1 / 2}}$,

where

$\lambda_{d e}=\left(\frac{\epsilon_{0} T_{e}}{N_{e} e^{2}}\right)^{1 / 2}$

is the electron Debye radius.

With regard for the collisions between electrons and dust particles, Eq. (11) for the effective collision frequency of electrons looks like

$\nu_{\text {eff }}=\nu_{e d}+\nu_{e i}+\nu_{e n}$. 


\subsection{Dielectric permittivity}

In a dusty plasma, the tensor of complex dielectric permittivity is determined by Eq. (3), in which the dielectric susceptibilities of the electron and ion plasma components, as well as dust particles, are taken into account $[9,11,29]$. In a number of works [30-33], their authors considered the transverse, $\varepsilon^{t r}(\omega, k)$, and longitudinal, $\varepsilon^{l}(\omega, k)$, components of the dielectric permittivity of a dusty plasma. In work [30], the following expressions were obtained for $\varepsilon^{l}(\omega, k)$ and $\varepsilon^{t r}(\omega, k):$

$$
\begin{aligned}
& \varepsilon^{l}(\omega, k)=1+\frac{4 \pi}{k^{2}} \sum_{\alpha} \frac{e_{\alpha}^{2}}{m_{\alpha}} \int d v \frac{1}{\omega-k \cdot v+i \nu_{\alpha d}} \times \\
& \times\left(1+\frac{i \nu_{\alpha d}}{\omega+i \nu_{c h}} \frac{1+\Gamma_{k \omega}^{(l)}}{1+G_{k \omega}}\right)\left(k \cdot \frac{d f_{\alpha}^{0}}{d v}\right), \\
& \varepsilon^{\operatorname{tr}}(\omega, k)=1+\frac{2 \pi}{\omega} \sum_{\alpha} \frac{e_{\alpha}^{2}}{m_{\alpha}} \int d v \frac{1}{\omega-k \cdot v+i \nu_{\alpha d}} \times \\
& \times\left(v_{i}+\frac{i \nu_{\alpha d}}{\omega+i \nu_{c h}} \frac{\Gamma_{k \omega, i}^{(t)}}{1+G_{k \omega}}\right)\left(\delta_{i j}-\frac{k_{i} k_{j}}{k^{2}}\right) \frac{d f_{\alpha}^{0}}{d v_{j}},
\end{aligned}
$$

where the quantities $\Gamma_{k \omega}^{(l)}, \Gamma_{k \omega, i}^{(t)}$, and $G_{k \omega}$ are determined by the expressions

$$
\begin{aligned}
\Gamma_{k \omega}^{(l)} & =\sum_{\alpha} \int d v \frac{-i e_{\alpha} \sigma_{\alpha} v f_{\alpha}^{0}}{\omega-k \cdot v+i \nu_{\alpha d}}, \\
\Gamma_{k \omega, i}^{(t)} & =\sum_{\alpha} \int d v \frac{-i e_{\alpha} \sigma_{\alpha} v v_{i} f_{\alpha}^{0}}{\omega-k \cdot v+i \nu_{\alpha d}}, \\
G_{k \omega} & =\frac{-1}{\omega+i \nu_{\mathrm{ch}}} \sum_{\alpha} \int d v \frac{\nu_{\alpha d} e_{\alpha} \sigma_{\alpha} v f_{\alpha}^{0}}{\omega-k \cdot v+i \nu_{\alpha d}} .
\end{aligned}
$$

For the Maxwell velocity distribution and at high frequencies $\omega \sim \omega_{p e} \gg \max \left(k v_{e}, \nu_{\mathrm{ch}}, \nu_{\alpha d}\right)$, expressions (31) and (32) for the longitudinal and transverse, respectively, dielectric permittivities coincide: $\varepsilon^{t r}(\omega, k)=\varepsilon^{l}(\omega, k)=\varepsilon^{\prime}(\omega)$, where $\varepsilon^{\prime}(\omega)$ is given by the expression

$\varepsilon^{\prime}=1-\frac{\omega_{p e}^{2}}{\omega^{2}}\left[1-i \frac{2}{3}(2+z) \frac{\nu_{e d}}{\omega}-\sqrt{\pi z} A\left(\frac{\nu_{e d}}{\omega}\right)^{2} \exp (z)\right]$

Here, $\tau_{T}=T_{i} / T_{e}$, and

$A(z)=\frac{5}{4}-\frac{z}{6}+\left(\frac{5}{4}-z+\frac{z^{2}}{3}\right) K(z)$, where

$K(z) \equiv \int_{1}^{\infty} d \tau_{T} \exp \left[-\left(\tau_{T}^{2}-1\right) z\right]$

In the case $\omega \gg k \nu_{e} \gg k \nu_{i}$, the authors of work [31] obtained the following expression for the complex dielectric permittivity:

$\varepsilon^{\prime}=1-\sum_{\alpha} \frac{\omega_{p \alpha}^{2}}{\omega^{2}}+\frac{i}{\omega^{3}} \sum_{\alpha} \omega_{p \alpha}^{2} v_{a d}$.

In works [33-35], analytic expressions were derived for the dielectric permittivity and the conductivity of a dusty plasma. The corresponding longitudinal and transverse dielectric permittivities of a dusty plasma are described by the expressions [33]:

$$
\begin{aligned}
& \varepsilon^{l}(\omega, k)=1+\left(1-\frac{i \omega N_{d}}{\omega+i \nu_{c h}} \frac{\sigma_{\alpha}\left(v_{\alpha}\right)}{k}\right) \times \\
& \times \frac{\omega_{p a}^{2}}{k^{2} v_{a}^{2}} \frac{W(\xi)}{1-\frac{i \nu_{\alpha n}}{\omega+i \nu_{\alpha n}}(1-W(\xi))}, \\
& \varepsilon^{t r}(\omega, k)=1-\left(1-\frac{i \omega N_{d}}{\omega+i \nu_{c h}} \frac{\sigma_{\alpha}\left(v_{\alpha}\right)}{k}\right) \times \\
& \times \frac{\omega_{p a}^{2}}{\omega+i \nu_{a n}^{2}} \frac{1-W(\xi)}{1-\frac{i \nu_{\alpha n}}{\omega+i \nu_{\alpha n}}(1-W(\xi))},
\end{aligned}
$$

where $\xi=\left(\omega+i \nu_{\alpha n}\right) /\left(k v_{\alpha}\right)$. For a cold plasma, $|\xi| \gg$ $\gg 1$, and the dependence $W(\xi)$ looks like

$W(\xi)=-\frac{k^{2} v_{\alpha}^{2}}{\left(\omega+i \nu_{a n}\right)^{2}}$.

In the case $|W(\xi)| \ll 1$, formulas (40) and (41) for the longitudinal and transverse dielectric permittivities coincide: $\varepsilon^{t r}(\omega, k)=\varepsilon^{l}(\omega, k)=\varepsilon^{\prime}(\omega)$, where $\varepsilon^{\prime}(\omega)$ is given by the expression

$\varepsilon^{\prime}=1-\frac{\omega_{p a}^{2}}{\omega\left(\omega+i \nu_{a n}\right)}+\frac{i \omega_{p \alpha}^{2} N_{d} \sigma_{\alpha}\left(v_{\alpha}\right)}{k\left(\omega+i \nu_{a n}\right)\left(\omega+i \nu_{\mathrm{ch}}\right)}$.

From expression (43), taking Eq. (5) for the complex dielectric permittivity into account, and neglecting the ionic component, we obtain the following formulas for the dielectric permittivity and the highfrequency conductivity in a dusty plasma:

$\varepsilon=1-\frac{N_{e} e^{2}}{\epsilon_{0} m_{e}\left(\omega^{2}+\nu_{\mathrm{eff}}^{2}\right)}+$ 
$+\frac{\omega}{k} \eta_{d} \frac{N_{e} e^{2}}{\epsilon_{0} m_{e}} \frac{\left(\nu_{\mathrm{eff}}+\nu_{\mathrm{ch}}\right)}{\left(\omega^{2}+\nu_{\mathrm{eff}}^{2}\right)\left(\omega^{2}+\nu_{\mathrm{ch}}^{2}\right)}$

$\sigma=\frac{N_{e} e^{2} \nu_{\mathrm{eff}}}{m_{e}\left(\omega^{2}+\nu_{\mathrm{eff}}^{2}\right)}+$

$+\frac{\omega}{k} \eta_{d} \frac{N_{e} e^{2}}{m_{e}} \frac{\omega^{2}-\nu_{\mathrm{eff}} \nu_{\mathrm{ch}}}{\left(\omega^{2}+\nu_{\mathrm{eff}}^{2}\right)\left(\omega^{2}+\nu_{\mathrm{ch}}^{2}\right)}$,

where $\nu_{\text {eff }}$ is the effective electron collision frequency, which is determined by Eq. (30), and the parameter $\eta_{d}=N_{d} \sigma_{e}\left(v_{e}\right)$.

\subsection{Refractive and absorption indices}

When considering the refractive, $n$, and absorption, $\varkappa$, indices in a dusty plasma, two approaches are
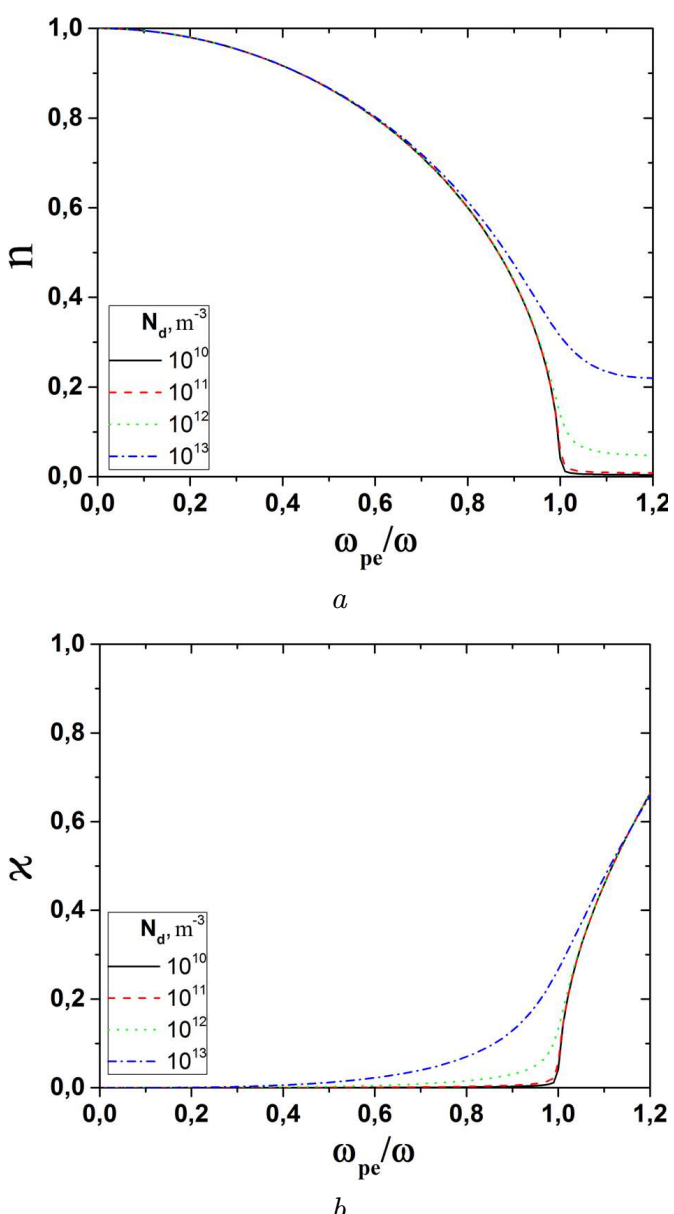

Fig. 2. Dependences of the refractive $(a)$ and absorption $(b)$ indices in a dusty plasma on the ratio $\omega_{p e} / \omega$ for various concentrations of dust particles. The calculation parameters are $r_{d}=1 \times 10^{-6} \mathrm{~m}, N_{i}=1 \times 10^{16} \mathrm{~m}^{-3}, N_{n}=1 \times 10^{20} \mathrm{~m}^{-3}$, $T_{e}=1 \mathrm{eV}$, and $T_{i}=0.1 T_{e}$ used. In one of them, expressions for $n$ and $\varkappa$ obtained for a particle-free plasma - see formulas (15), (16), (19), and (20) - are used [12,23,36-38]. In this case, the transition to a dusty plasma is performed, by considering the additional frequencies of particle collisions with dust particles (see Section 2.1). This approach is rather justified and can be used in the case of high frequencies, which follows from Eq. (39) and a small number of particles in plasma.

The results of calculations of the refractive, $n$, and absorption, $\varkappa$, indices obtained according to formulas (15) and (16) for weakly ionized and strongly ionized dusty plasmas are exhibited in Figs. 2 to 4. The effective collision frequency was determined from Eq. (30). The calculations were carried out with regard for the following conditions:

- Ar ions were assumed to be single-charged;

- the rate constant for electron-neutral collisions was calculated, by supposing the Maxwell distribution of electrons over their velocities and taking the cross-sections of elastic collisions into account [39];

- the $\mathrm{Cu}_{2} \mathrm{O}$ dust particles were assumed to be perfect spheres of the same radius;

- the charge of dust particles was determined by equality (26);

- the quasineutrality condition (23) for a dusty plasma was supposed to be obeyed.

As one can see from Figs. 2 and 4, an increase of the dust particle concentration induces the growth of both the refractive, $n$, and absorption, $\varkappa$, indices. This is a result of an increase of the parameter $\nu_{\text {eff }}$. In essence, the observed scenario is similar to that discussed in Section 2.2 (see Fig. 1). However, in this case, an important factor is the collision frequency of electrons with dust particles. An increase of the dust particle size changes the values ofboth $n$ and $\varkappa$, as is shown in Fig. 3. Thus, the concentration and the size of dust particles substantially affect the refractive and absorption indices, especially if $\omega^{2} \rightarrow \omega_{p e}^{2}$. In the high-frequency limit, when $\omega^{2} \gg \omega_{p e}^{2}$ and $\omega^{2} \gg \nu_{\text {eff }}^{2}$, the influence of dust particles on $n$ and $\varkappa$ can be neglected in some cases.

In the other approach, besides the frequency of collisions between particles and dust particles, the characteristic frequency of the dust particle charging is also taken into account. In this case, expression (44) for the dielectric permittivity and expression (45) for the high-frequency conductivity of a dusty plasma are 

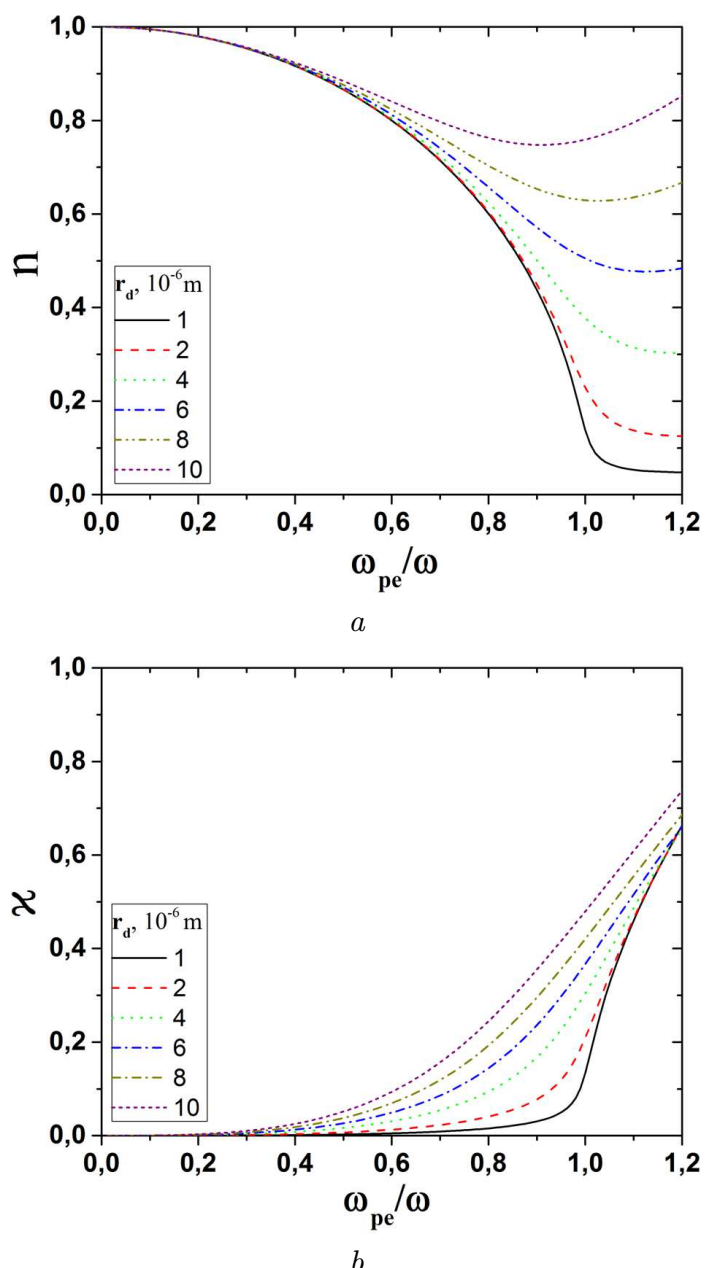

Fig. 3. Dependences of the refractive $(a)$ and absorption $(b)$ indices in a dusty plasma on the ratio $\omega_{p e} / \omega$ for various values of the dusty particle radius. The calculation parameters are $N_{d}=10^{12} \mathrm{~m}^{-3}, N_{i}=1 \times 10^{16} \mathrm{~m}^{-3}, N_{n}=1 \times 10^{20} \mathrm{~m}^{-3}$, $T_{e}=1 \mathrm{eV}$, and $T_{i}=0.1 T_{e}$

used. The formulas for the refractive index $n$ and the absorption index $\varkappa$ can be written as follows [33]:

$n=\left(\frac{1}{2} \varepsilon+\frac{1}{2} \sqrt{\varepsilon^{2}+\left(\frac{\sigma}{\omega \epsilon_{0}}\right)^{2}}\right)^{1 / 2}$,

$\varkappa=\left(-\frac{1}{2} \varepsilon+\frac{1}{2} \sqrt{\varepsilon^{2}+\left(\frac{\sigma}{\omega \epsilon_{0}}\right)^{2}}\right)^{1 / 2}$,

where $\varepsilon$ is determined by expression (44) and $\sigma$ by expression (45). This approach [33-35] was applied in ISSN 2071-0194. Ukr. J. Phys. 2019. Vol. 64, No. 5
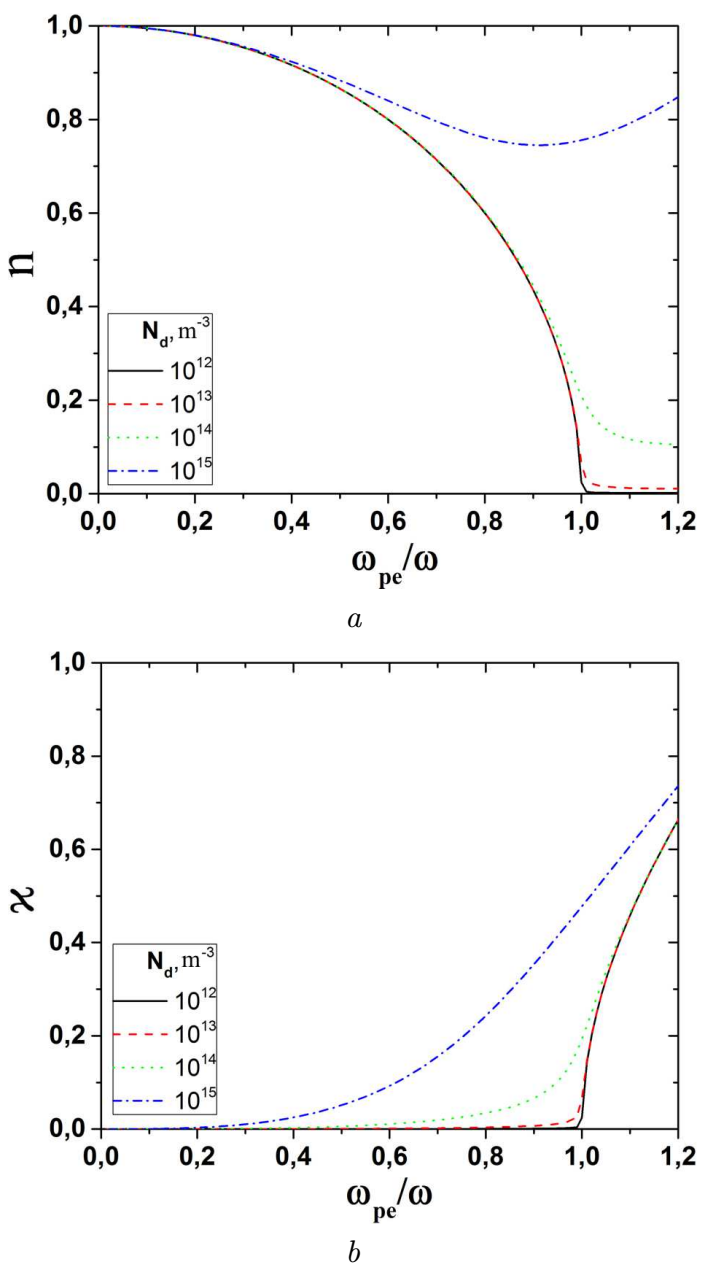

Fig. 4. Dependences of the refractive $(a)$ and absorption (b) indices in a dusty plasma on the ratio $\omega_{p e} / \omega$ for various concentrations of dust particles. The calculation parameters are $r_{d}=1 \times 10^{-6} \mathrm{~m}, N_{i}=1 \times 10^{19} \mathrm{~m}^{-3}, N_{n}=1 \times 10^{19} \mathrm{~m}^{-3}$, $T_{e}=10 \mathrm{eV}$ and $T_{i}=0.1 T_{e}$

a number of works [24, 40-51]. In so doing, a number of simplifications and assumptions in Eqs. (43)-(46) are often made $[24,33-35,40-51]$.

First - see, e.g., works [33-35,40-43,45-50]) - when calculating the parameter $\eta_{d}$, it is assumed that the absorption cross-section $\sigma_{e}\left(v_{e}\right) \approx \pi r_{d}^{2}$, so that this quantity depends only on the dust particle size. In works [24, 44], expression (24) was used, where the potential of the dust particle surface is determined by the formula $\phi_{s}=\frac{Z_{d} e}{4 \pi \epsilon_{0} r_{d}}$. Accordingly, Eq. (24) takes the form

$\sigma_{e}\left(\nu_{e}\right)=\pi r_{d}^{2}\left(1+\frac{Z_{d} e^{2}}{6 \pi \epsilon_{0} r_{d} T_{e}}\right)$ 

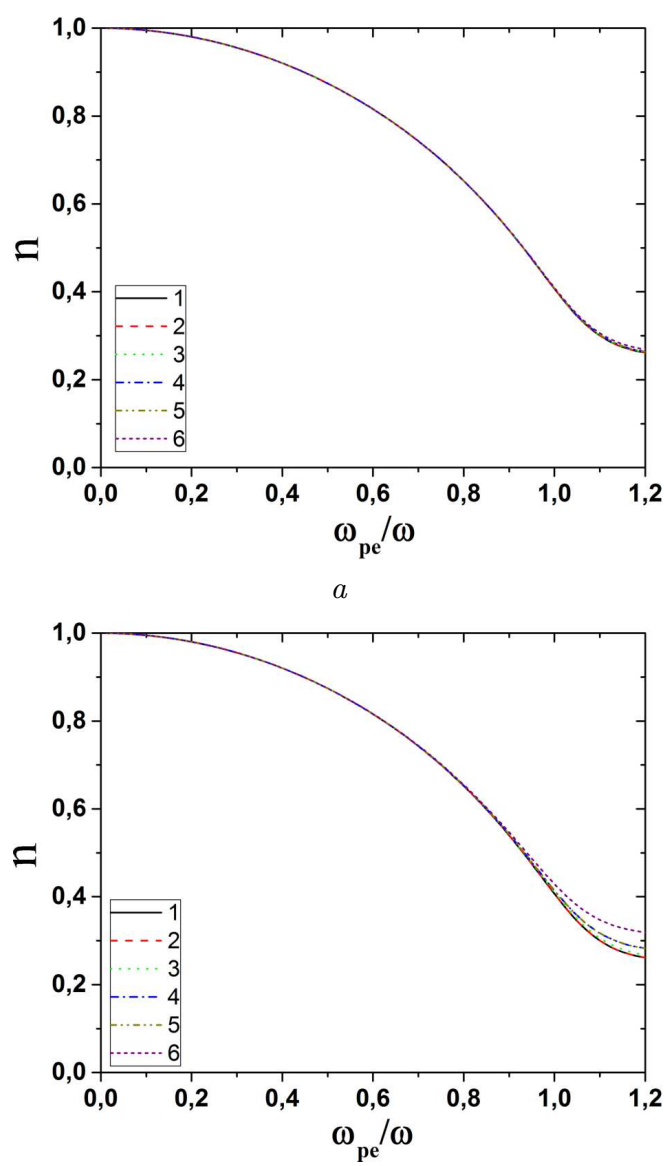

$b$

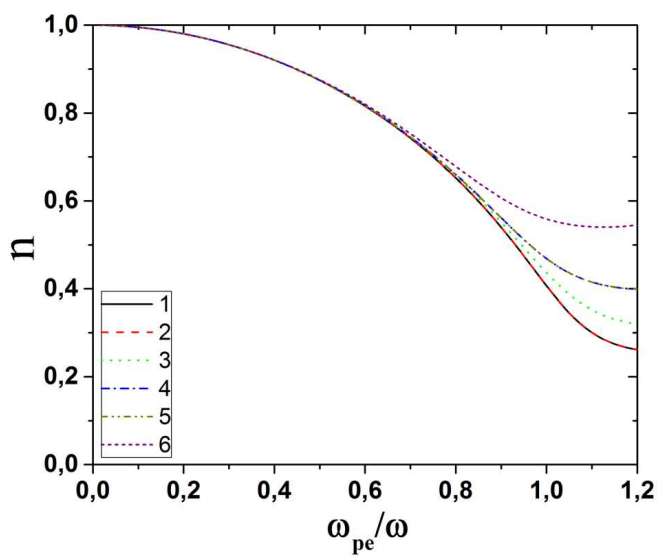

Fig. 5. Dependences of the refractive index $n$ in a dusty plasma on the ratio $\omega_{p e} / \omega$ in various approximations (on the curve labeling from 1 to 6 , see the text). The parameter $N_{d}=10^{11}(a), 10^{12}(b)$, and $10^{13} \mathrm{~m}^{-3}(c)$. The other calculation parameters are $r_{d}=1 \times 10^{-6} \mathrm{~m}, N_{i}=1 \times 10^{19} \mathrm{~m}^{-3}$, $N_{n}=10^{24} \mathrm{~m}^{-3}, T_{e}=3000 \mathrm{~K}$, and $T_{i}=0.1 \times T_{e}$

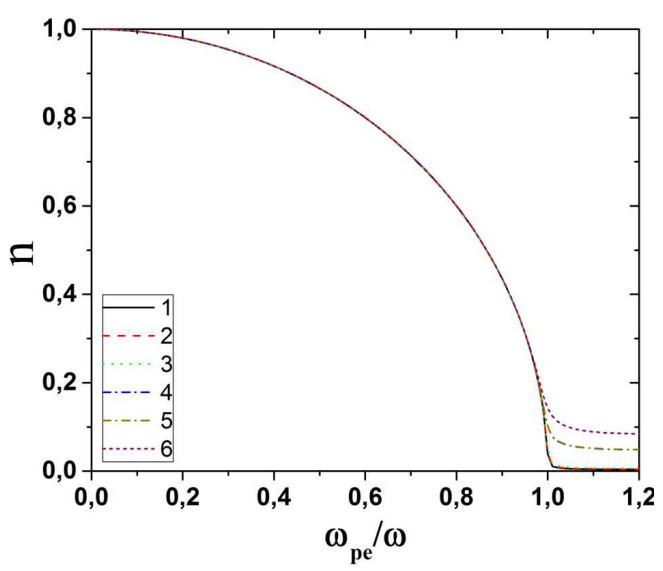

$a$

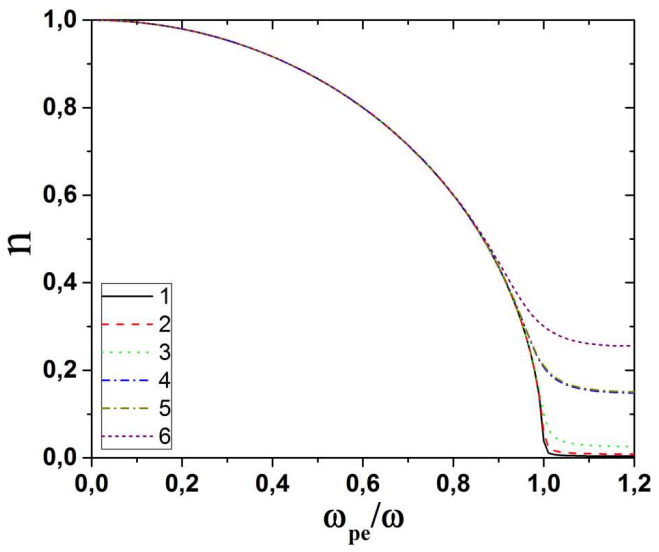

$b$

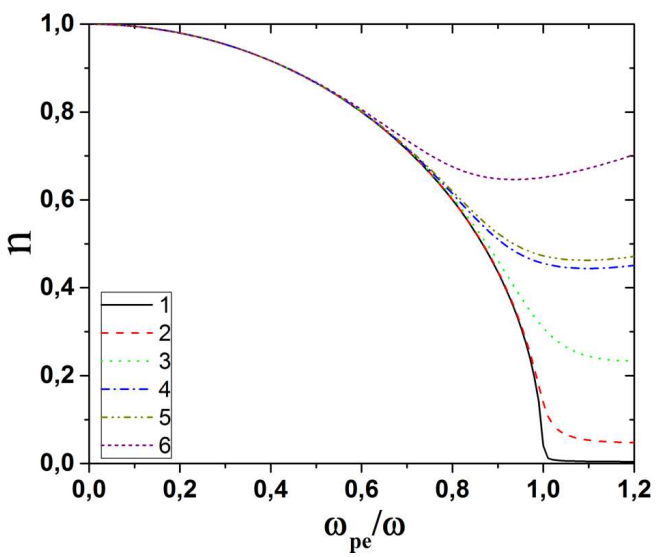

Fig. 6. Dependences of the refractive index $n$ in a dusty plasma on the ratio $\omega_{p e} / \omega$ in various approximations (on the curve labeling from 1 to 6 , see the text). The parameter $N_{d}=10^{10}(a), 10^{11}(b)$, and $10^{12} \mathrm{~m}^{-3}(c)$. The other calculation parameters are $r_{d}=1 \times 10^{-6} \mathrm{~m}, N_{i}=1 \times 10^{16} \mathrm{~m}^{-3}$, $N_{n}=10^{20} \mathrm{~m}^{-3}, T_{e}=1 \mathrm{eV}$, and $T_{i}=0.1 \times T_{e}$

ISSN 2071-0194. Ukr. J. Phys. 2019. Vol. 64, No. 5 
It allows the dependence of the absorption crosssection on the electron temperature, as well as the size and charge of dust particles, to be taken into account more accurately.

Second, the quantity $\omega / k$ in Eqs. (44) and (45), which is actually the parameter $\nu_{p h}=\omega / k$ in the medium, is put equal to the light velocity in vacuum, $\nu_{p h}=c$ (see, e.g., works [33-35, 40,41, 43-51]). This substitution is quite allowable, if the refractive index $n \approx 1$. But, in the case $n \neq 1$, the refractive index has to be taken into account: $\omega / k=c / n$. Then Eqs. (44)(47) can be solved numerically, while determining $n$ and $\varkappa$.

Since different approximations are used in calculations, it is of interest to compare the refractive index values obtained from Eqs. (44)-(47) and from Eqs. (15), (16), (19), and (20). The conditions already described above were chosen as initial ones: Ar ions, $\mathrm{Cu}_{2} \mathrm{O}$ particles, and so forth. The plasma parameters were taken according to the data of work [46] and to the parameters of weakly ionized plasma, which were also described above (see the caption of Fig. 2). The calculation results obtained for the refractive index are presented in Figs. 5 and 6. The labeling of the curves in those figures are as follows. Curves 1 and 2 were calculated by formula (15): curves 1 not taking $\nu_{e d}$ into account and curves 2 taking $\nu_{e d}$ into account. Curves 3 to 6 were calculated according to formulas (44)-(46): curve 3 for $\omega / k=c$, $\sigma_{e}\left(v_{e}\right) \approx \pi r_{d}^{2}$, and not taking $\nu_{e d}$ into account; curve 4 for $\omega / k=c / n, \sigma_{e}\left(v_{e}\right) \approx \pi r_{d}^{2}$, and not taking $\nu_{e d}$ into account; curve 5 for $\omega / k=c / n, \sigma_{e}\left(v_{e}\right) \approx \pi r_{d}^{2}$, and taking $\nu_{e d}$ into account; and curve 6 for $\omega / k=c / n$, $\sigma_{e}\left(v_{e}\right)$ calculated by formula (48), and not taking $\nu_{e d}$ into account.

As one can see from Figs. 5, $a$ and $6, a$, at $\omega^{2} \gtrsim \omega_{p e}^{2}$ and a relatively low concentration of particles, the calculated values of $n$ are almost identical for all approximations and approaches. With the increase of the dust particle concentration (Figs. 5, b, 5, c, 6, b, and $6, c)$, the calculation results begin to differ from one another in different approaches and approximations starting from $\omega_{p e} / \omega \gtrsim 0.5 \div 0.6$. The calculated values of $n$ are maximum in the case where Eqs. (44)-(47) were used. The minimum values were obtained, when no allowance was made for dust particles - Eqs. (11), (15), and (16) - which is quite reasonable. A similar situation also takes place, when the size of dust particles is varied. The analysis testifies that, in the

ISSN 2071-0194. Ukr. J. Phys. 2019. Vol. 64, No. 5 high-frequency limit, i.e. at $\omega^{2} \gg \omega_{p e}^{2}$ and $\omega^{2} \gg \nu_{\text {eff }}^{2}$, the influence of dust particles on $n$ and $\varkappa$ can be neglected sometimes. In those cases, Eqs. (19)-(22) can be used for calculations. Hence, the density, size, and charge of dust particles substantially affect the refractive and absorption indices of a dusty plasma.

Besides those dust particle parameters, the refractive and absorption indices also depend on the probing frequency (see Figs. 3 to 6 ). Therefore, the measurements of the phase shift and the absorption coefficient at various frequencies can be used to evaluate or determine such dusty plasma parameters as the average density, dust particle size, and frequency of collisions between electrons and dust particles.

For the picture to be complete, it is also worth noting the following two specific features of a dusty plasma. First, the charge of dust particles can be rather large. Accordingly, the concentration of ions can substantially exceed the concentration of electrons. In this case, the ionic component in the dielectric permittivity has to be taken into account (see, e.g., Eqs. (7) and (8)). Second, in the general case, dust particles are different by their size and are described by a certain distribution function $[9,12]$. In works $[34,35,43,45]$, for this size distribution to be taken into consideration, expressions (44) and (45) were proposed to be used in the form of the sums

$\varepsilon=\sum_{j=1}^{N_{d}} \varepsilon_{j}$ and $\sigma=\sum_{j=1}^{N_{d}} \sigma_{j}$

over dust particle groups with identical sizes, or this summation should be carried out only over the parameter $\eta_{d}[43,45]$ :

$\eta_{d}=\sum_{j=1}^{N_{d}} N_{d j} \pi r_{d j}^{2}$

The analysis of the high-frequency conductivity in a dusty plasma with various model distributions of dust particles over their size showed [43] that this parameter can increase or decrease, depending on the distribution itself and on the difference between the maximum and minimum particle sizes.

\section{Conclusions}

The refractive, $n$, and absorption, $\varkappa$, indices in weakly and strongly ionized dusty plasmas are calculated and compared in the framework of various 
approaches and approximations. If the concentration of dust particles is low, and if the frequency is high, $\omega^{2} \geq \omega_{p e}^{2}$, the calculated values of $n$ practically coincide in all approximations and approaches. At higher concentrations of dust particles, the calculation results obtained in different approaches and approximations begin to deviate from one another at $\omega_{p e} / \omega \geq 0.5 \div 0.6$. A similar situation also takes place for the varied dust particle size. Hence, the concentration and size of dust particles significantly affect the refractive and absorption indices for microwaves, especially if $\omega^{2} \rightarrow \omega_{p e}^{2}$. In the high-frequency limit, i.e. when $\omega^{2} \gg \omega_{p e}^{2}$ and $\omega^{2} \gg \nu_{\text {eff }}^{2}$, the influence of dust particles on $n$ and $\varkappa$ can be neglected in some cases, so that methods developed for a dustfree plasma can be applied. The measurements of the phase shift and the absorption coefficient at various frequencies can provide some information about such parameters of a dusty plasma as the average density, dust particle size, and frequency of collisions between electrons and dust particles.

1. M.A. Heald, C.B. Wharton. Plasma Diagnostics with Microwaves (Wiley, 1965).

2. V.E. Golant. Superhigh-Frequency Methods of Plasma Research (Nauka, 1968) (in Russian).

3. H.-J. Hartfuss, T. Geist. Fusion Plasma Diagnostics with mm-Waves: An Introduction (Wiley, 2013) [ISBN: 978-3527-41105-4].

4. E. Mazzucato. Electromagnetic Waves for Thermonuclear Fusion Research (World Scientific, 2014) [ISBN: 978-9814571-80-7].

5. C. Killer, T. Wegner, A. Melzer, J. Meichsner. Influence of dust particles on the bulk electron density in radio frequency plasmas measured by microwave interferometry. Phys. Plasmas 22, 123702 (2015).

6. Th. Wegner, A.M. Hinz, F. Faupel, T. Strunskus, H. Kersten, J. Meichsner. Influence of nanoparticle formation on discharge properties in argon-acetylene capacitively coupled radio frequency plasmas. Appl. Phys. Lett. 108, 063108 (2016).

7. Jieshu Jia, Chengxun Yuan, Ruilin Gao, Zhong-Xiang, Zhou, Ying Wang, Xiaoou Wang, Hui Li, Hui Li, Jian Wu. Propagation of electromagnetic waves in a glow-discharge dusty plasma. In: Proceedinds of the 11th International Symposium on Antennas, Propagation and EM Theory (2016), p. 198.

8. Jieshu Jia, Chengxun Yuan, Ruilin Gao, Sha Liu, Feng Yue, Ying Wang, Zhong-Xiang Zhou, Jian Wu, Hui Li. Transmission characteristics of microwave in a glow-discharge dusty plasma. Phys. Plasmas 23, 073705 (2016).
9. P.K. Shukla, A.A. Mamun. Introduction to Dusty Plasma Physics (CRC Press, 2001) [ISBN: 9780750306539].

10. V.N. Tsytovich, G.E. Morfill, S.V. Vladimirov, H.M. Thomas. Elementary Physics of Complex Plasmas (SpringerVerlag, 2008) [ISBN: 3540290001].

11. V.E. Fortov, G.E. Morfill. Complex and Dusty Plasmas: From Laboratory to Space (Taylor and Francis, 2010) [ISBN: 978-1-4200-8311-8].

12. M.S. Sodha. Kinetics of Complex Plasmas (Springer, 2014) [ISBN: 978-81-322-1819-7].

13. O. Bystrenko, A. Zagorodny. Screening of dust grains in a weakly ionized gas: Effects of charging by plasma currents. Phys. Rev. E 67, 066403, (2003).

14. I.L. Semenov, A.G. Zagorodny, I.V. Krivtsun. Ion drag force on a dust grain in a weakly ionized collisional plasma. Phys. Plasmas 20, 013701 (2013).

15. A.I. Momot, A.G. Zagorodny, I.S. Orel. Interaction force between two finite-size charged particles in weakly ionized plasma. Phys. Rev. E 95, 013212 (2017).

16. V.N. Tsytovich, U. de Angelis, R. Bingham. Transition scattering of waves on charged dust particles in a plasma. J. Plasma Phys. 42, 429 (1989).

17. U. de Angelis, R. Bingham, V.N. Tsytovich, Dispersion properties of dusty plasmas. J. Plasma Phys. 42, 445 (1989).

18. R. Bingham, U. de Angelis, V.N. Tsytovich, O. Havnes. Electromagnetic wave scattering in dusty plasmas. Phys. Plasmas B 3, 811 (1991).

19. V.N. Tsytovich. Scattering of waves in dusty plasmas. Phys. Scripta 45, 521 (1992).

20. U. de Angelis. The physics of dusty plasmas. Phys. Scripta 45, 465 (1992).

21. C. La Hoz. Radar scattering from dusty plasmas. Phys. Scripta 45, 529 (1992).

22. O. Havnes, F. Melandsca, C. La Hoz, T.K. Aslaksen. Charged dust in the Earth's mesopause; Effects on radar backscatter. Phys. Scripta 45, 535 (1992).

23. M.S. Sodha, S.K. Mishra, Sh. Misra. Nonlinear dependence of complex plasma parameters on applied electric field. Phys. Plasmas 18, 023701 (2011).

24. Jieshu Jia, Chengxun Yuan, Sha Liu, Feng Yue, Ruilin Gao, Ying Wang, Zhong-Xiang Zhou, Jian Wu, Hui Li, Propagation of electromagnetic waves in a weak collisional and fully ionized dusty plasma. Phys. Plasmas 23, 043302 (2016).

25. A.I. Akhiezer, I.A. Akhiezer, R.V. Polovin, A.G. Sitenko, K.N. Stepanov. Plasma Electrodynamics. Vol. 1: Linear Theory (Pergamon Press, 1975).

26. A.I. Akhiezer, I.A. Akhiezer, R.V. Polovin, A.G. Sitenko, K.N. Stepanov. Plasma Electrodynamics. Vol. 2: Nonlinear Theory and Fluctuations (Pergamon Press, 1975).

27. V.L. Ginzburg. Propagation of Electromagnetic Waves in Plasma (Gordon and Breach, 1961).

28. S.A. Khrapak, G.E. Morfill. Dusty plasmas in a constant electric field: Role of the electron drag force. Phys. Rev. E 69, 066411 (2004).

ISSN 2071-0194. Ukr. J. Phys. 2019. Vol. 64, No. 5 
29. A.G. Sitenko, A.G. Zagorodny, Yu.I. Chutov, P. Schram, V.N. Tsytovich, Statistical properties and relaxation of dusty plasmas. Plasma Phys. Control. Fusion 38, A105 (1996).

30. S.V. Vladimirov. Propagation of waves in dusty plasmas with variable charges on dust particles. Phys. Plasmas 1, 2762 (1994).

31. S.A. Trigger, P.P.J.M. Schram. Kinetic theory of the charging process in dusty plasmas. J. Phys. D 32, 234 (1999).

32. Hui Li, Jian Wu, Zhong-Xiang Zhou, Chengxun Yuan, Jieshu Jia. The dielectric function of weakly ionized dusty plasmas. Phys. Plasmas 23, 073301 (2016).

33. Shi Yan-Xiang, Wu Jian, Ge De-Biao. The research on the dielectric tensor of weakly ionized dust plasma. Acta Phys. Sin. 58, 5507 (2009).

34. Shi Yan-Xiang, Ge De-Biao, Wu Jian. Influence of charge and discharge processes of dust particles on the dust plasma conductivity. Acta Phys. Sin. 55, 5318 (2006).

35. Shi Yan-Xiang, Ge De-Biao, Wu Jian. Theoretical analysis of microwave attenuation constant of weakly ionized dusty plasma. Chin. J. Geophys. 50, 877 (2007).

36. Hui Li, Jian Wu, Zhongxiang Zhou, Chengxun Yuan. Propagation of electromagnetic wave in dusty plasma and the influence of dust size distribution. Phys. Plasmas 23 073702 (2016)

37. Hui Li, Jian Wu, Chengxun Yuan, Zhongxiang Zhou. The electrical conductivity of weakly ionized plasma containing dust particles. Phys. Lett. A 380, 2540 (2016).

38. Yunhai Hong, Chengxun Yuan, Jieshu Jia, Ruilin Gao, Ying Wang, Zhongxiang Zhou, Xiaoou Wang, Hui Li, Jian Wu. Propagation characteristics of microwaves in dusty plasmas with multi-collisions. Plasma Sci. Technol. 19, 055301 (2017).

39. R. Mayol, F. Salvat. Total and transport cross sections for elastic scattering of electrons by atoms. At. Data Nucl. Data Tables 65, 55 (1997).

40. Yan Xiang Shi, Hui Min Chen, P. Zumaji. Studying the electromagnetic property of dust plasma in polar mesospheric during summer. In: Proceedings of the 9th International Symposium on Antennas Propagation and EM Theory (2010), p. 383.

41. Hui Li, Jian Wu, Yan-Xiang She, Jun Wu. Microwave attenuation characteristics of the dusty plasma produced by solid rocket exhausts. In: Proceedings of the 9th International Symposium on Antennas Propagation and EM Theory (2010), p. 371.

42. Li Lin-Qian, Shi Yan-Xiang, Wang Fei, Wei Bing. SOFDTD method of analyzing the reflection and transmission coefficient of weakly ionized dusty plasma layer. Acta Phys. Sin. 61, 125201 (2012).

43. Ji-Zheng Duan, Cang-Long Wang, Jian-Rong Zhang, Sheng-Qian Ma, Xue-Ren Hong, Jian-An Sun, WenShan Duan, Lei Yang. Influence of charging process and size distribution of dust grains on the electric conductivity of dusty plasma. Phys. Plasmas 19, 083703 (2012).
44. Chen Yun-Yun, Zheng Gai-Ge, Gu Fang, Li Zhen-Hua. Effect of dust particle potential on plasma conductivity. Acta Phys. Sin. 61, 154202 (2012).

45. Ji-Zheng Duan, Juan-Fang Han, Cang-Long Wang, YanXia Xu, Jian-Rong Zhang, Wen-Shan Duan, Lei Yang. Contribution of the dust grains to the damping of the electromagnetic waves propagating in plasma. IEEE Trans. Plasma Science 41, 2434 (2013).

46. Jieshu Jia, Chengxun Yuan, Ruilin Gao, Ying Wang, Yaoze Liu, Junying Gao, Zhongxiang Zhou, Xiudong Sun, Jian Wu, Hui Li, Shaozhi Pu. Propagation of electromagnetic waves in a weakly ionized dusty plasma. J. Phys. D 48, 465201 (2015).

47. Maoyan Wang, Mengxia Yu, Zhitao Xu, Guiping Li, Baojun Jiang, Jun $\mathrm{Xu}$. Propagation properties of terahertz waves in a time-varying dusty plasma slab using FDTD. IEEE Trans. Plasma Science 43, (2015).

48. Maoyan Wang, Zhitao Xu, Yuliang Dong, Jun Xu, Meng Zhang. The interaction of terahertz waves and a dusty plasma slab with Epstein distribution. Frequenz $\mathbf{7 0}$, 39 (2016).

49. Maoyan Wang, Hailong Li, Yuliang Dong, Guiping Li, Baojun Jiang, Qiang Zhao, Jun Xu. Propagation matrix method study on $\mathrm{THz}$ waves propagation in a dusty plasma sheath. IEEE Trans. Antennas and Propagation 64, 286 (2016).

50. Wang Maoyan, Zhang Meng, Li Guiping, Jiang Baojun, Zhang Xiaochuan, Xu Jun. FDTD simulation on terahertz waves propagation through a dusty plasma. Plasma Sci. Technol. 18, 798 (2016).

51. Yun-yun Chen, Fang Gu, Cui-hong Yang, Yingying Zhang. The study of dusty plasma's conductivity with dual-temperature model. Optik 127, 2476 (2016).

Received 09.12.17.

Translated from Ukrainian by O.I. Voitenko

Ю.В. Ковтун, А.І. Скибенко,

Є.I. Скібенко, С.В. Сюсъко

ОСОБЛИВОСТІ ВИКОРИСТАННЯ

МІКРОХВИЛЬОВИХ МЕТОДІВ ДЛЯ ДІАГНОСТИКИ

ЗАПОРОШЕНОЇ ПЛАЗМИ. І. ДІЕЛЕКТРИЧНА

ПРОНИКНІСТЬ, ПОКАЗНИКИ

ЗАЛОМЛЕННЯ ТА ПОГЛИНАННЯ

Р е $з$ ю м е

Проаналізовано два підходи, які зазвичай використовуються для визначення показників заломлення $n$ і поглинання $\varkappa$ запорошеної плазми. У першому використовують вирази для $n$ i $\varkappa$, отримані для плазми без порошинок з урахуванням додаткових частот зіткнень частинок 3 порошинками. У другому враховуються не тільки частоти зіткнень частинок з порошинками, а і характерна частота зарядки порошинок 